\title{
Performance Analysis of AODV Protocol on MANET's Model with ViSim
}

\author{
K.V. Satyanarayana ${ }^{1}$ and K.Sudha ${ }^{2}$ \\ ${ }^{1}$ Department of Computer Science and Engineering Avanthi Institute of Engineering \\ \& Technology, Visakhapatnam, India \\ ${ }^{2}$ Department of Computer Science and Engineering Lenora College of Engineering, \\ AP, India \\ ${ }^{1}$ thanqji@gmail.com
}

\begin{abstract}
The usage of the MANET network models had increased a lot in the recent years due to various advantages of these networks. The major advantage was the size of the model and the network topology. The network of these models changes from time to time due to the reason that the addition of nodes and separation of nodes to the network model is always possible and very frequent in these network models. Hence, it is very important and required to analyze the performance of these networks with the help of various routing protocols. The performance had analyzed with various performance metrics like the end to end delay of the network model, output of the network etc. The results are displayed and discussed in detail in the results section.
\end{abstract}

Keywords: Quality of Service (QoS) metrics, DSR, ViSim

\section{Introduction}

In MANETs (Mobile Ad-hoc Networks) performance is sensitive to quality, quantifiability and traffic load etc, [1][2][3]. Examining completely different routing protocols performance in dynamic surroundings plays a significant role in economical routing. Here in my default parameters of DSR routing protocol in MANET's for locating the impact in QoS Metrics that area unit taking part in a vital role within the performance of associate degree infrastructureless network [4]. And conjointly finding the performance varied configurations in routing protocols of MANETs in conjunction with varied default various parameters results like QoS parameters (throughput, delay, etc.). During this paper, the most contribution is to investigate the performance of reactive routing protocol in ad-hoc networks beneath completely different eventualities supported different parameters by mistreatment and ViSim machine [5][6]. Mistreatment ViSim machine by varied QoS parameters and analyzing the performance of DSR reactive routing protocol [7][8].

\section{Routing protocols in MANET'S}

\subsection{Wireless links}

The foremost necessary purpose to be thought-about whenever we have a tendency to be employing a wireless network particularly all the nodes within the device network are operating

Article history:

Received (April 6, 2019), Review Result (June 12, 2019), Accepted (September 21, 2019) 
below wireless mode. Because of the nodes are connected within the mode of a wireless mode, all the nodes during a wireless network are ready to simply be attacked by different attackers within the network. Wired networks cannot get to be conducted or to be issued because the nodes don't need the physical affiliation to access the info from the nodes and therefore the attacks on the nodes within the network. Hence, for mistreatment of the wireless networks the information measure ought to get to be minimum.

\subsection{Dynamic topology}

The nodes within the mobile Adhoc networks are within the dynamic in nature in terms of the topology and their operations. The topology of the network and therefore the nodes that were connected within the network may amendment from time to time. Because of the nodes within the wireless network changes from time to time, the topology of the network changes and the quantity of the load on the network and behavior of the nodes within the network and therefore the performance of the network also changes as results of the consequences that were being taken place. Because of the topology and therefore the position of the nodes within the network changes from time to time, the attacks on the nodes may additionally be magnified during a sensible variety.

\section{Simulation tool using ViSim}

ViSim is one of the principally used simulators within the field of analysis and within the trade. The most reasons for the illustrious of this toll are thanks to its use-friendly program so the users will use simple and can also build the changes to the prevailing network mixtures[1][5]. As a result of these facilities, the ViSim could be useful for researchers, students, lecturers in their individual analysis work, and for the expression of a range of wireless network eventualities on pc screens and its connected user output devices. The usage of this machine had become the task of simulation for varied devices had created the items and also the machine models become terribly illustrious and extremely simple. The interest of the users for utilizing this machine had adult lots within the customers and users for this machine in of late [8].

\subsection{Implementation of ViSim Scenario}

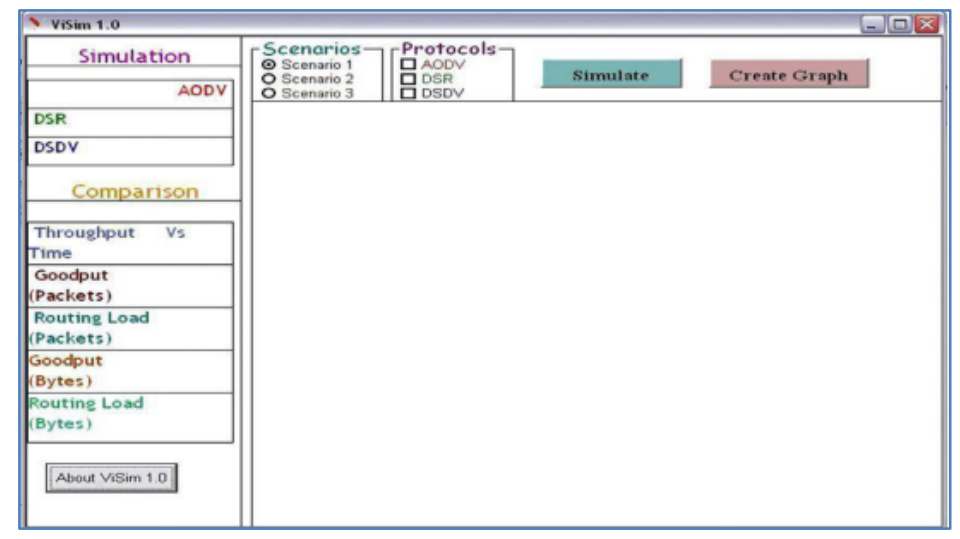

Figure 1. Selection of scenarios in the user interface of the simulator

In [Figure 1], the selection of various and other related things can be selected by using or creating the different scenarios with various constraints. Various scenarios with the 
combination of several protocols in the network with various network performance metrics were selected from the above given [Figure 1].

\subsection{Functional examples}

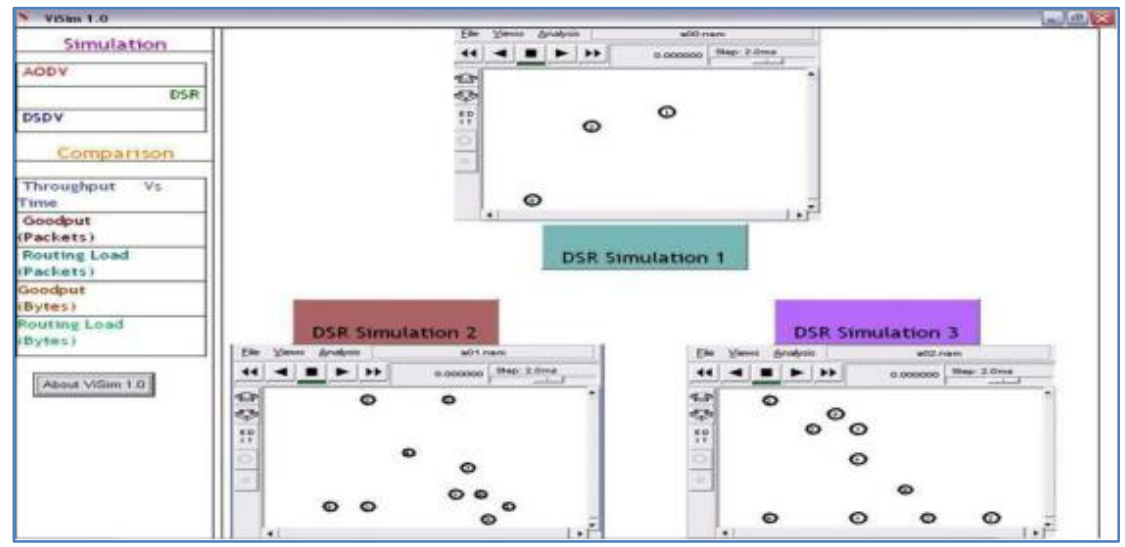

Figure 2. Creation of two scenarios in the user interface of the simulator

In [Figure 2], the three sets of nodes were selected and a two network models with two scenarios were created by using the simulator with the combination of the AODV protocol. All the three network models were working with the help of the AODV protocol.

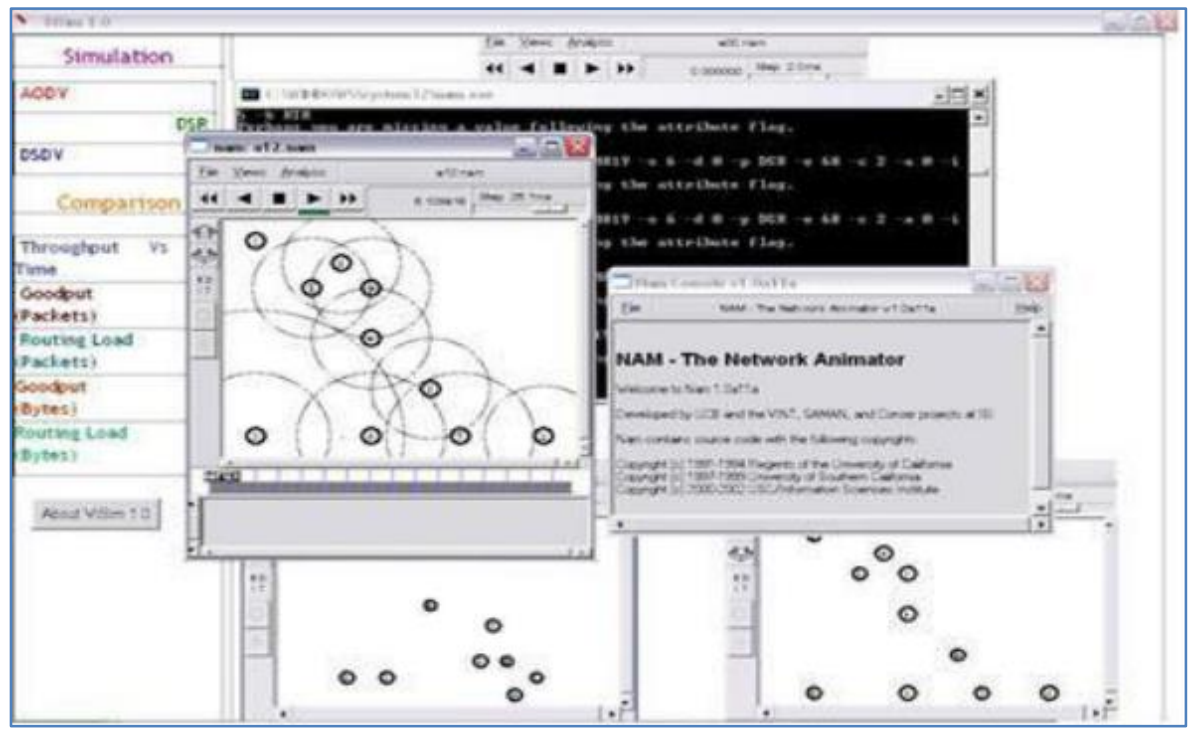

Figure 3. The output after choosing AODV simulation

From [Figure 3], the creation of several nodes in the assumed network with various loads considerations and their configurations with their loads of input and output of the nodes were also discussed so far. The figure gives a brief idea of creating the nods and their programming model for the working and executing the process of the network model was also to be considered and implemented. 


\section{Simulation of the 2 scenarios}

\subsection{Scenario-1 (5 nodes)}

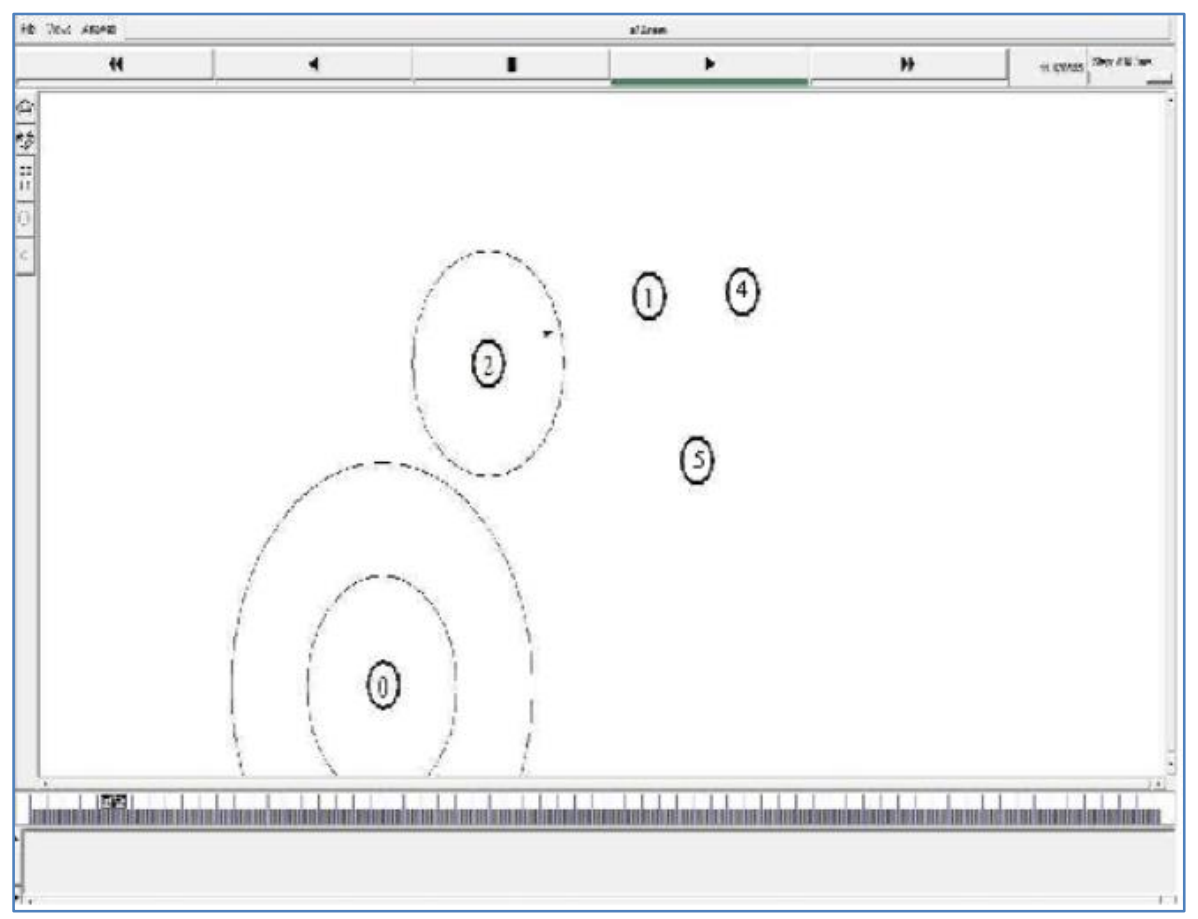

Figure 4. The creation of five nodes in the scenario 1

In [Figure 4], the creation of the three nodes in the simulator model was displayed.

\subsection{Scenario-2 (15 nodes)}

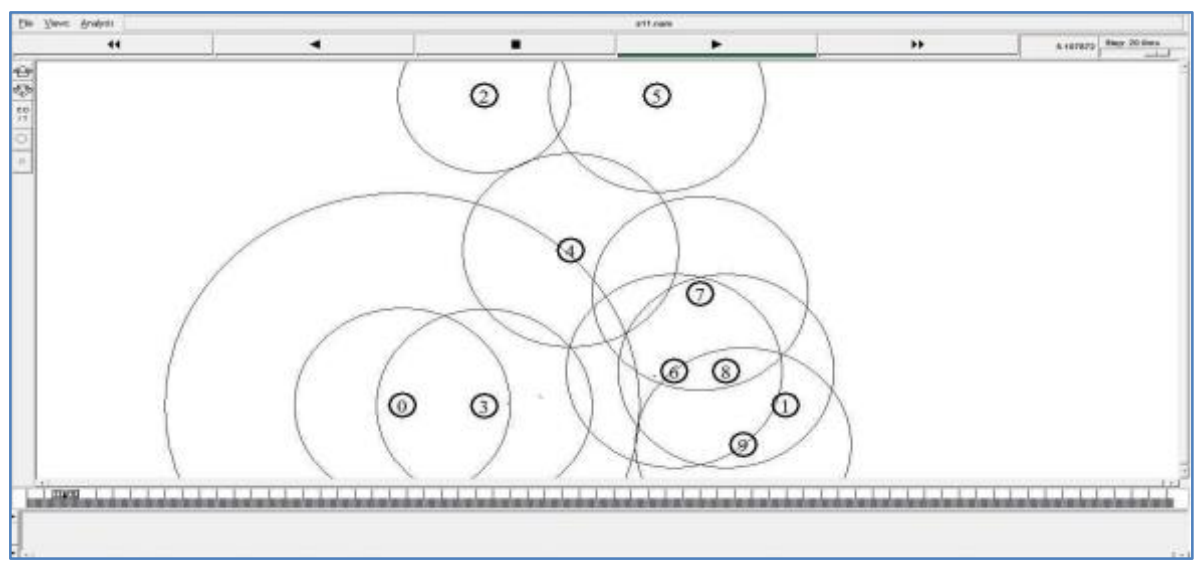

Figure 5. The creation of fifteen nodes in the scenario 2

In [Figure 5], the simulator was selected such that to create a set of fifteen nodes in the wireless network as the scenario 2 . 


\subsection{Throughput of AODV at 5,15}

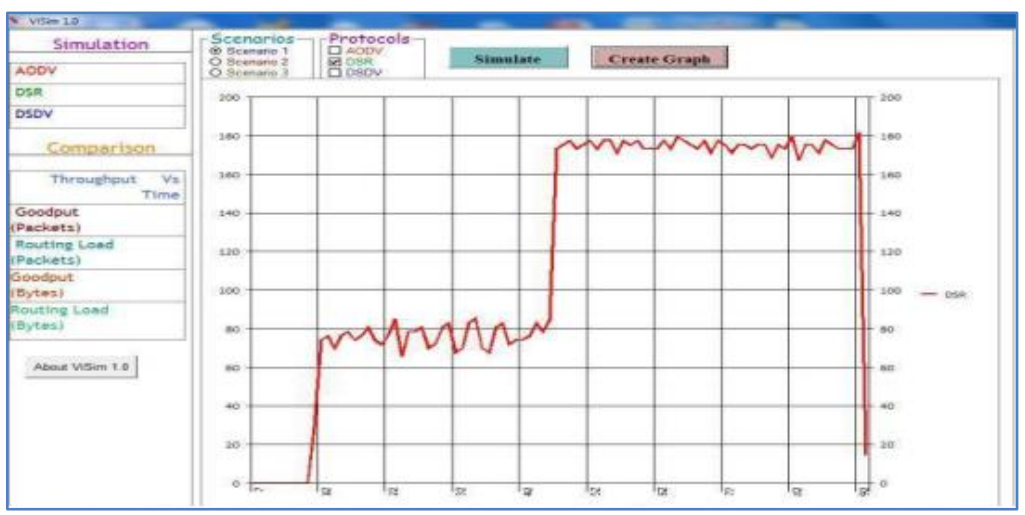

Figure 6. The throughput of five nodes in the scenario 1

In [Figure 6], the simulator was selected such that the throughput of the network with three set nodes at scenario 1 was studied and represented.

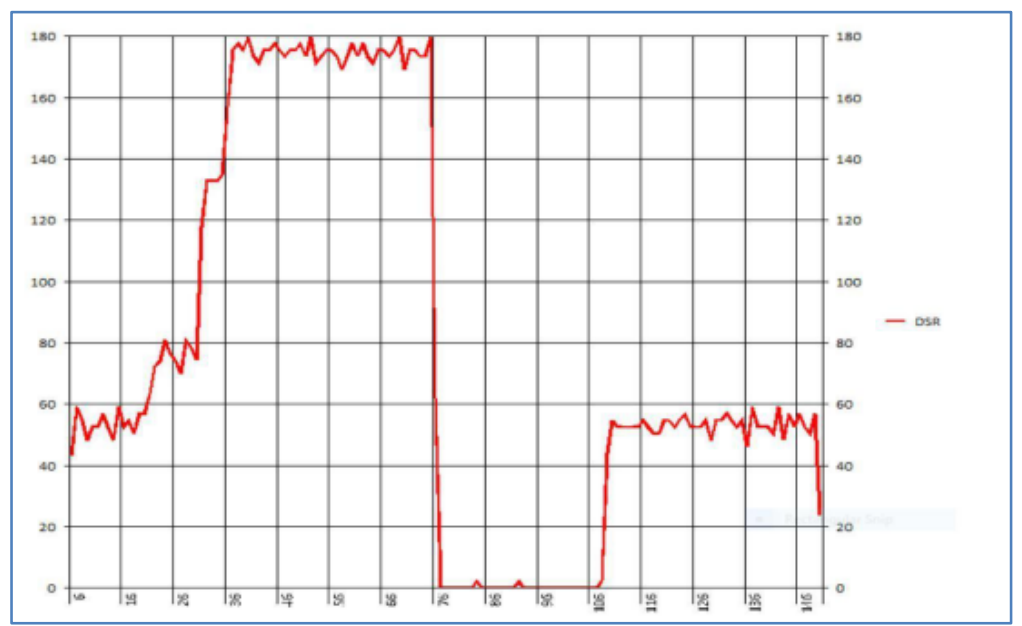

Figure 7. The throughput of fifteen nodes in the scenario 2

In [Figure 7], the simulator was selected such that the throughput of the network with fifteen nodes at scenario 2 was studied and represented.

\subsection{Routing load packets at 5, 15 nodes}

In [Figure 8], the simulator was selected such that the routing load on the network was analyzed with the help of the graphical representation. The number of nodes that were selected is the five nodes in the network.

In [Figure 9], the simulator was selected such that the routing load on the network was analyzed with the help of the graphical representation. The number of nodes that were selected is the fifteen nodes in the network. 


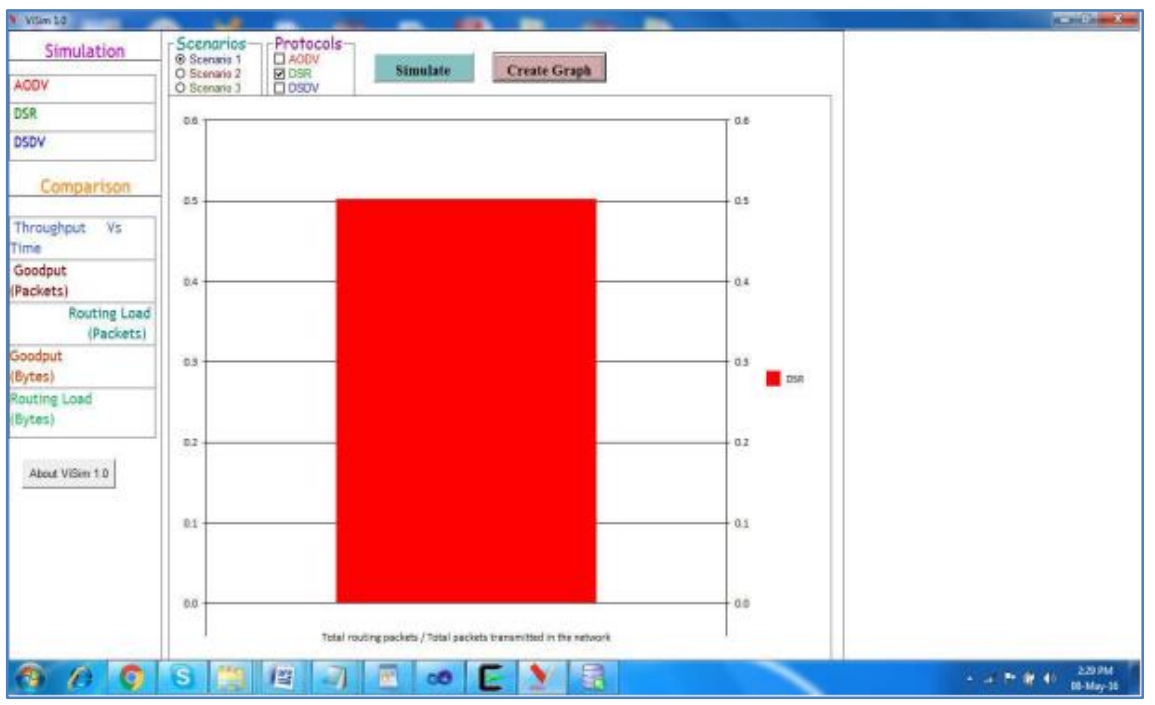

Figure 8 . The routing load on the packets for five nodes in the scenario 1

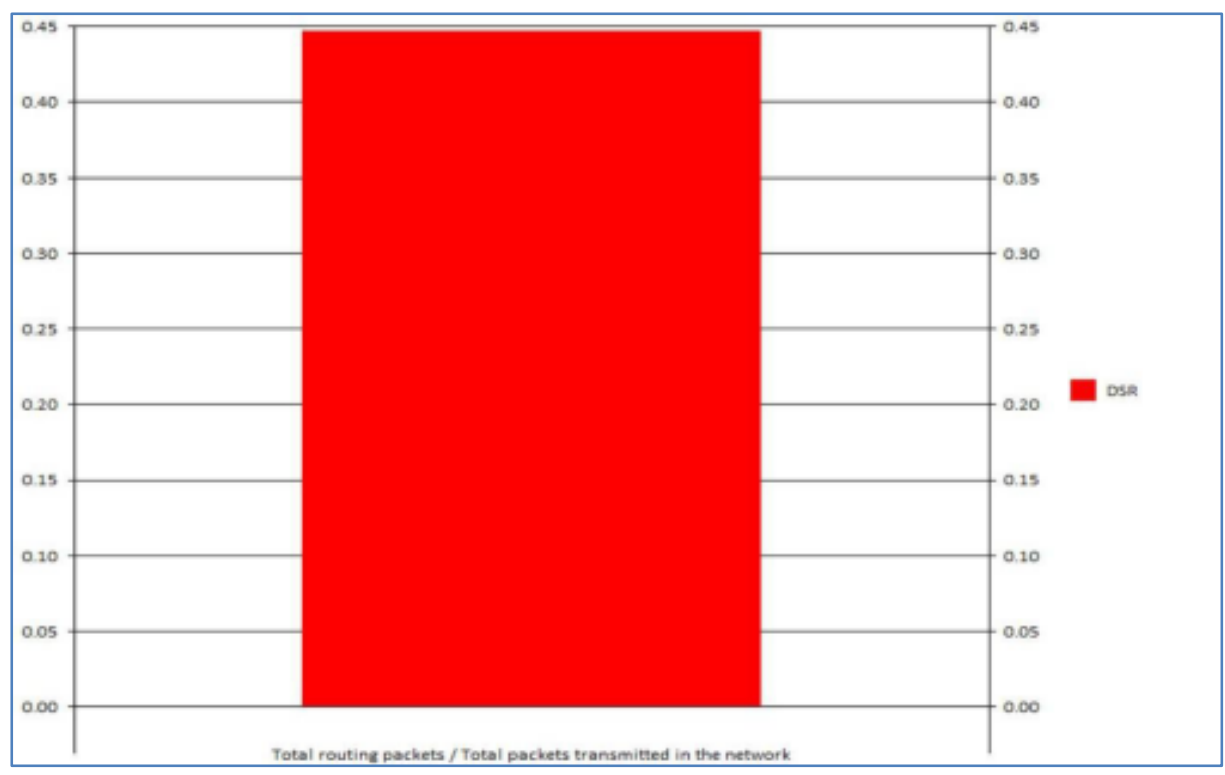

Figure 9. The routing load on the packets fifteen nodes in the scenario 2

\subsection{Routing load bytes at 5, 15 nodes}

In [Figure 10], the simulator was selected such that the routing load in terms of bytes on the network was analyzed with the help of the graphical representation. The number of nodes that were selected is the five nodes in the network.

In [Figure 11], the simulator was selected such that the routing load in terms of bytes on the network was analyzed with the help of the graphical representation. The number of nodes that were selected is the fifteen nodes in the network. 


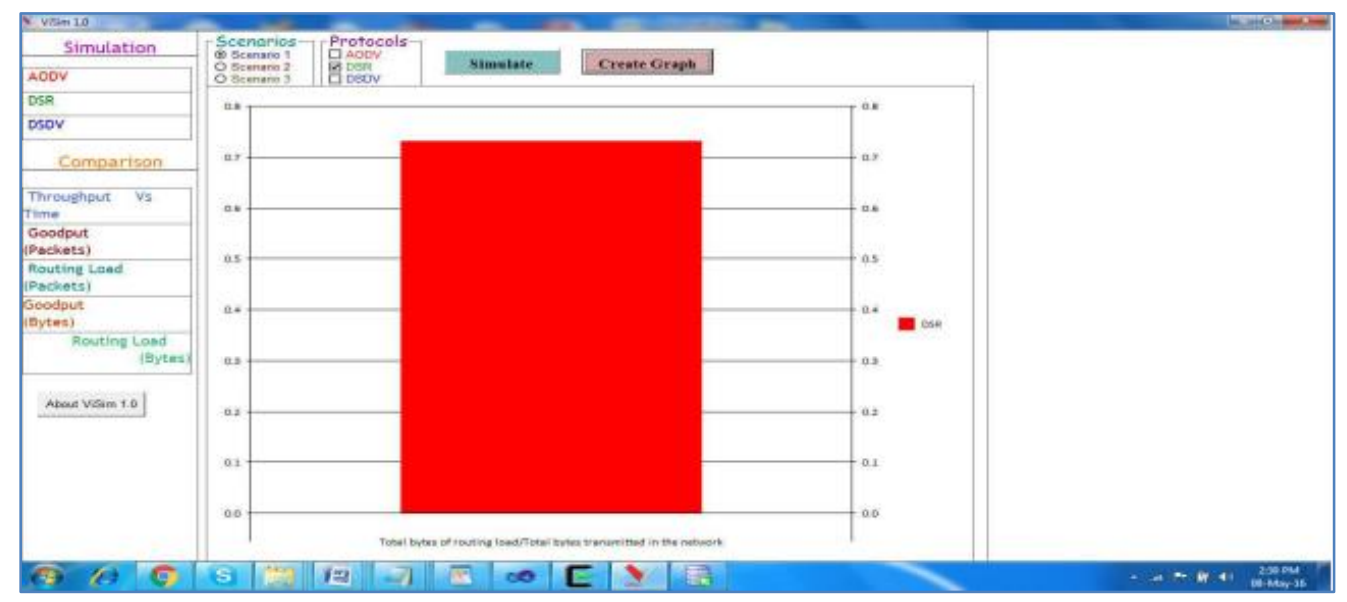

Figure 10. The routing load on the packets five nodes in the scenario 1

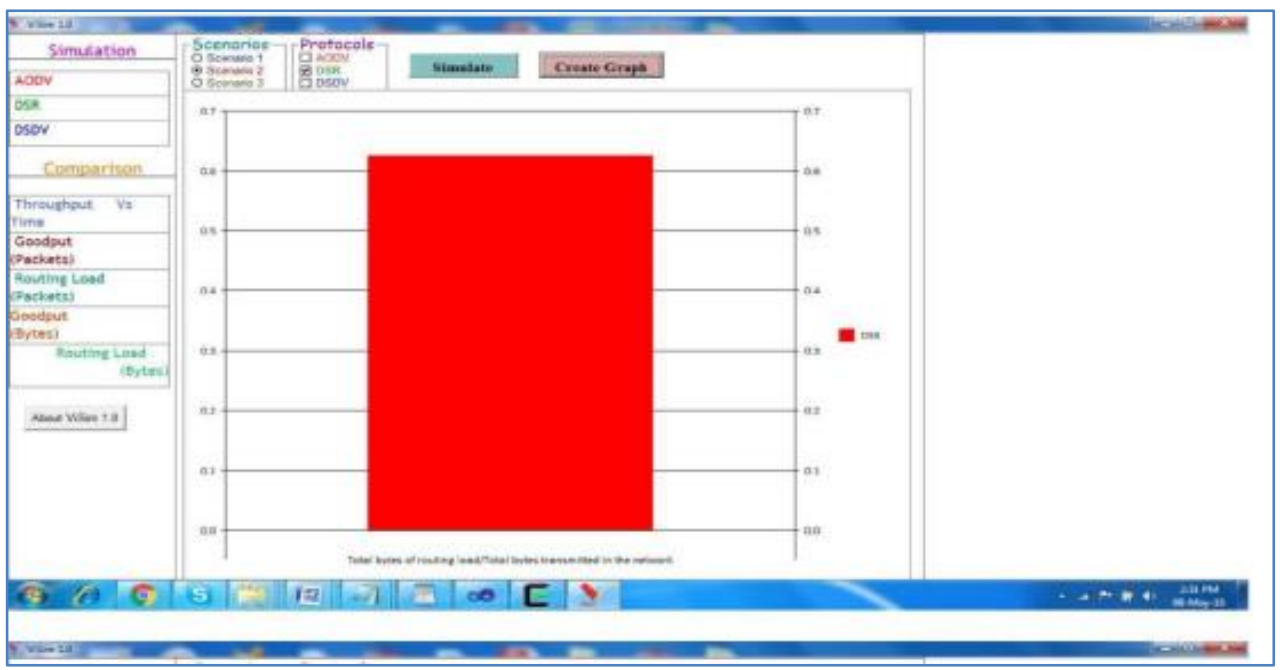

Figure 11. The routing load on the packets fifteen nodes in the scenario 2

\section{Conclusion}

In this paper, the performance of the wireless sensing element network with varied figures of nodes within the network at varied mixtures known as varied situations were mentioned and bestowed. The simulation tool ViSim was used with success and total performance of the network at varied conditions and also the performance metrics of the networks were analyzed with the assistance of graphical representations. The performance metrics of the communication networks just like the outturn of the nodes within the network at various levels like the 5 nodes within the network, 15 nodes within the network. The results and also the performance were with success diagrammatic by victimization the graphical illustration. The performance of the network with the employment of AODV protocol within the network for knowledge transmissions was studied with success with the mix of 2 sets of nodes in every state of affairs. 


\section{References}

[1] N.Thirupathi Rao, G. Sudeepthi, and Debnath Bhattacharyya, "Studies on the performance evaluation of protocols in MANETs using simulation," International Journal of Future Generation Communication and Networking, 2019, vol.12, no.2, pp.71-80, (2019).

[2] Erciyes, Kayhan, Dagdeviren, Orhan, Cokuslu, Deniz, Yilmaz, Onur, and Gumus, Hasan, "Modeling and simulation of mobile Ad hoc networks," Mobile Ad Hoc Networks, pp.134-168, (2011) DOI: 10.1201/b114475

[3] N.Thirupathi Rao, P. Srinivas, Debnath Bhattacharyya, and Tai-hoon Kim, "A detailed review on mobile ad hoc networks: Protocols, security issues and challenges," International Journal of Security and Its Applications, (ESCI, Web of Science), vol.12, no.2, pp.67-78, (2018)

[4] TÜNCEL, Sinan \& EKİ, Hüseyin, and Zengin, Ahmet, "Design and implementation of a new MANET simulator model for AODV simulation," Turkish Journal of Electrical Engineering \& Computer Sciences, vol.24, no.4, pp.2239-2254, (2016) DOI: 10.3906/elk-1311-120

[5] N.Thirupathi Rao, Debnath Bhattacharyya, and Hye-Jin Kim, "Analyzing the increase of network life time for wireless sensor network with smart local moving algorithm using NS2 simulator," International Journal of Future Generation Communication and Networking, vol.10, no.9, pp.75-84, (2017) DOI: 10.14257/ijfgen.2017.10.9.07

[6] Stuart Kurkowsk, Tracy Camp, and Michael Colagrosso, "MANET simulation studies: The incredibles," Mobile Computing and Communications Review, vol.9, no.4, pp.50-61, (2016) DOI: 10.1145/1096166.1096174

[7] N.Thirupathi Rao, Debnath Bhattacharyya, V. Madhusudana Rao, and Tai-hoon Kim, "Analyzing the Behaviour of Reactive Protocols in Wireless Sensor Networks Using TCP Mode of Connection,” International Journal of Engineering and Advanced Technology (IJEAT), vol.8, no.4, pp.1387-1392, (2019)

[8] Luc Hogie, Pascal Bouvry, Frederic Guinand, “An overview of MANETs simulation," Electronic Notes in Theoretical Computer Science, vol.150, pp.81-101, (2006) DOI:10.1016/j.entcs.2005.12.025 